\title{
Pretargeting with bispecific fusion proteins facilitates delivery of nanoparticles to tumor cells with distinct surface antigens
}

\author{
Qi Yang ${ }^{a}$, Christina L. Parker ${ }^{a}$, Yukang Lin ${ }^{b}$, Oliver W. Press ${ }^{b}$, Steven I. Park ${ }^{c}$, Samuel K. Lai ${ }^{a, d, e *}$ \\ a'Division of Pharmacoengineering and Molecular Pharmaceutics, Eshelman School of Pharmacy, \\ University of North Carolina at Chapel Hill; ${ }^{\text {}}$ The Fred Hutchinson Cancer Research Center, Seattle, WA \\ and the Department of Medicine at the University of Washington, ${ }^{\mathrm{c} D i v i s i o n}$ of Hematology and \\ Oncology, School of Medicine, University of North Carolina at Chapel Hill,; ${ }^{\mathrm{d}}$ UNC/NCSU Joint \\ Department of Biomedical Engineering; ${ }^{e}$ Department of Microbiology \& Immunology; University of \\ North Carolina at Chapel Hill, Chapel Hill, NC 27599
}

Running title: Pretargeted delivery of nanoparticles to distinct tumors

Keywords: drug delivery, tumor heterogeneity, lymphoma, streptavidin/biotin, ligand density

\author{
*Corresponding authors: \\ Samuel K. Lai \\ Division of Molecular Pharmaceutics \\ University of North Carolina at Chapel Hill \\ Marsico 4213, 125 Mason Farm Road \\ Phone: (+001)-919-966-3024 \\ E-mail: lai@unc.edu \\ Homepage: http://www.lailab.com \\ Steven I. Park \\ Lymphoma Program/Division of Hematology and Oncology \\ School of Medicine \\ University of North Carolina at Chapel Hill \\ 170 Manning Drive, CB\#7305 \\ Phone: 919-843-6616 \\ E-mail: sipark@med.unc.edu
}




\begin{abstract}
Tumor heterogeneity, which describes the genetically and phenotypically distinct subpopulations of tumor cells present within the same tumor or patient, presents a major challenge to targeted delivery of diagnostic and/or therapeutic agents. An ideal targeting strategy should deliver the same nanocarriers to the full diversity of cancer cells, which is difficult to achieve with conventional ligand-conjugated nanoparticles. We evaluated pretargeting (i.e., multistep targeting) as a strategy to facilitate nanoparticle delivery to target cells by measuring the uptake of biotinylated nanoparticles by lymphoma cells with distinct surface antigens pretreated with bispecific streptavidin-scFv fusion proteins. Fusion proteins targeting CD20 or tumor-associated glycoprotein (TAG) 72 mediated the specific uptake of $100 \mathrm{~nm}$ biotin-functionalized nanoparticles by Raji (CD20-positive B-cell) and Jurkat (TAG72-positive T-cell) lymphoma cells, respectively. Greater uptake was observed for nanoparticles with increasing amounts of surface biotin, and fully biotin-modified particles remained resistant to cultured macrophage cell uptake, although they were still quickly cleared from systemic circulation in vivo. For single Raji-tumor bearing mice, pretargeting with CD20-specific FP significantly increased nanoparticle tumor targeting, and, in mice bearing both Raji and Jurkat tumors, pretargeting with both fusion proteins markedly increased nanoparticle targeting to both tumor types, compared to animals dosed with nanoparticles alone. These in vitro and in vivo observations support further evaluations of pretargeting fusion protein cocktails as a strategy to enhance nanoparticle delivery to a diverse array of molecularly distinct target cells.
\end{abstract}




\section{Introduction}

Effective delivery of therapeutic drug molecules or imaging contrast agents to cancer cells continues to be one of the biggest challenges in cancer therapy and diagnostics. Strategies used to target nanoparticles to cancer cells generally exploit unique features of tumor physiology such as (1) the poorly formed and leaky blood vessels that facilitate enhanced nanoparticle extravasation from the tumor vasculature relative to normal vasculature (i.e., the Enhaced Permeation and Retention [EPR] effect), and (2) the differential expression of surface receptors relative to normal tissues $[1,2]$. Unfortunately, the extent of the EPR effect varies substantially among different tumors and different subjects [3, 4]. "Stealth" nanoparticles that rely solely on "passive targeting" via the EPR effect are also poorly internalized, limiting the efficiency of intracellular delivery $[2,5]$. Although, "actively targeted" nanoparticles with tumor-specific ligands can theoretically bind and internalize into specific tumor cells, they are often quickly eliminated from the circulation due to rapid mononuclear phagocyte system (MPS) clearance [6]; this can result in a far lower fraction of the administered dose of ligand-conjugated nanoparticles extravasating at target tissue(s) relative to passively targeted nanoparticles. Indeed, in numerous reports in the literature, actively targeted nanoparticle systems did not appreciably improve nanoparticle delivery to tumors when compared to passively targeted nanoparticles [7-9].

The aforementioned challenges are further exacerbated by tumor heterogeneity, which encompasses the functional and phenotypic differences between cancer cells such as cellular morphology, gene and protein expression, metabolism, motility, proliferation, level of drug resistance, and metastatic potential. Tumor heterogeneity can be caused by variations in accumulated genetic mutations, along with alterations in the local tumor microenvironment, that frequently lead to genomically distinct subclonal populations within the same tumor or between tumor lesions $[10,11]$. In turn, the existence of diverse cancer cell populations poses a major challenge to targeted delivery of diagnostic and/or therapeutic agents. Single ligandconjugated nanoparticles generally fail to facilitate delivery to the diverse range of cells present in a given tumor lesion or patient [8]. Tumor heterogeneity can also result in variable EPR effects within different 
regions of the same tumor. As a result, treatment with nanomedicines only leads to partial suppression of cancers, leading to eventual tumor regrowth and/or the development of drug-resistant tumors. A potential solution involves the conjugation of multiple ligands onto a single nanoparticle. Unfortunately, such an approach would not only incur exorbitant developmental costs but also require conjugation of excess targeting ligands that would likely result in rapid phagocytic clearance of the modified particles. Most current nanoparticle-based drug delivery strategies fail to deliver adequate therapeutic payload to the full spectrum of tumor cells present in a given patient.

A recently emerged strategy to facilitate targeted delivery of therapeutics is "pretargeting," which involves first introducing pretargeting molecules that can specifically bind the tumor cells of interest and then capture the therapeutic and/or diagnostic payload administered in a second injection (Figure 1). Pretargeting in the form of pretargeted radioimmunotherapy (PRIT) has been extensively studied in vivo and in human subjects to improve the delivery of radioactive payload relative to antibody-radiolabel drug conjugates [12] and has generally shown greater tumor specificity and safety compared to conventional radioimmunotherapy [13]. Despite the considerable success of PRIT, pretargeting has rarely been applied to improve drug delivery by polymeric nanoparticles. Consequently, the potential applications, limitations, and challenges of this targeting strategy remain largely unknown [14]. A particular attractive feature of pretargeting is the theoretical ease in increasing the breadth of nanoparticle targeting to heterogeneous and molecularly distinct tumors simply by tuning the combination of pretargeting molecules used. For a given pretargeting molecule, the tumor-binding domain can be modified, while the nanoparticle-binding domain remains unchanged (Figure 1). In this study, we sought to evaluate whether pretargeting with two different pretargeting bispecific fusion proteins (FPs) consisting of single-chain variable fragments (scFvs) linked to streptavidin (SA), which have been successfully used in PRIT $[15,16]$, can enhance the delivery of the same biotinylated polymeric nanoparticles simultaneously to corresponding T- and B-lymphoma cells in vitro and in vivo. 


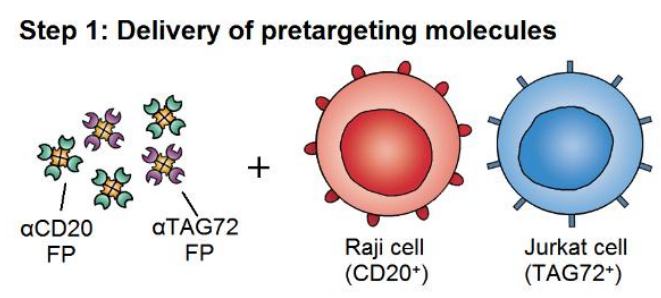

Step 2: Delivery of nanoparticle payload

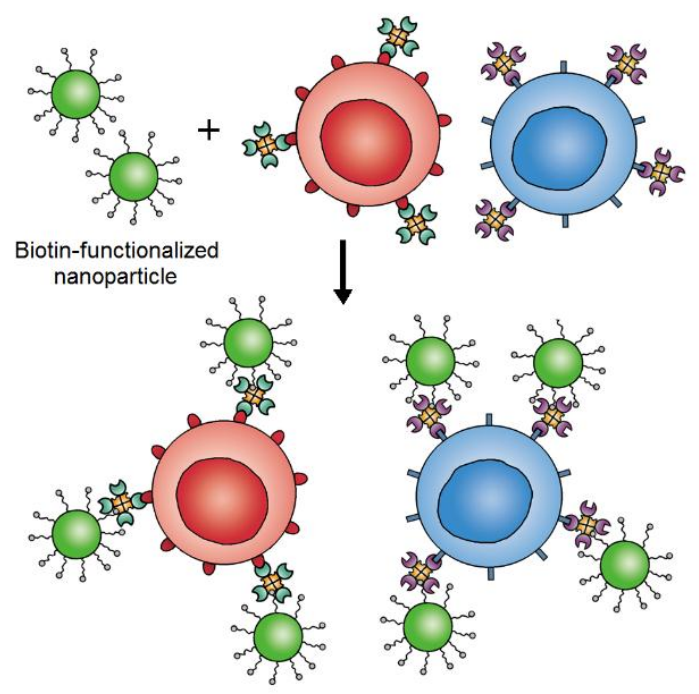

Figure 1. Schematic illustration of pretargeted nanoparticle delivery to a heterogeneous population of lymphoma cells. In the first step, a cocktail of pretargeting fusion proteins (FPs), based on streptavidin linked to single-chain variable fragments, is used to specifically label Raji B-cell lymphoma $\left(\mathrm{CD} 20^{+}\right)$and Jurkat T-cell lymphoma $\left(\mathrm{TAG} 2^{+}\right.$) cells. In the second step, biotin-functionalized nanoparticles are administered and will be captured by FPs bound to the surface of target cells.

\section{Materials and Methods}

\subsection{Preparation and characterization of PS-PEG-biotin nanoparticles}

Carboxylate-modified yellow-green fluorescent polystyrene (PS) beads with mean diameters of $\sim 100 \mathrm{~nm}$ were purchased from Invitrogen (Carlsbad, CA, USA). The surface COOH densities of the particles (3.4 $\mathrm{COOH}$ groups $/ \mathrm{nm}^{2}$ ) were calculated from the $\mathrm{mEq} / \mathrm{g}$ values provided by the manufacturer. Methoxy PEG amine (mPEG-NH 2,3 and $5 \mathrm{kDa}$ ) was purchased from JenKem (Allen, TX, USA), and biotin PEG amine (bPEG-NH $\mathrm{N}_{2}, 3 \mathrm{kDa}$ ) was obtained from Rapp Polymere (Tuebingen, Germany). Amine-modified PEGs were conjugated to the PS particles as previously described [17]. Briefly, the beads were washed thrice with MilliQ $\mathrm{H}_{2} \mathrm{O}$ and resuspended in $50 \mathrm{mM}$ borate buffer (pH 7.8). $\mathrm{mPEG}-\mathrm{NH}_{2}$ and bPEG- $\mathrm{NH}_{2}$ were 
added to the PS beads at varying methoxy PEG:biotin PEG ratios, and 1-ethyl-3-(3-

dimethylaminopropyl)carbodiimide (EDC; Invitrogen) and N-hydroxysulfosuccinimide (S-NHS; Thermo Scientific, MA) were each added at five-fold molar excess of total PEG amine. The EDC/S-NHS reaction was allowed to proceed overnight at RT. The reaction mixture was quenched with excess glycine, and the PEG-modified particles were washed with MilliQ $\mathrm{H}_{2} \mathrm{O}$ and resuspended in water to stock concentrations $(\sim 20 \mathrm{mg} / \mathrm{mL})$. The hydrodynamic size and $\zeta$-potential of the synthesized particles were determined by dynamic light scattering and laser Doppler anemometry, respectively, using a Zetasizer Nano ZS (Malvern, UK). The final PEG grafting density was indirected quantified using a 1pyrenylyldiazomethane (PDAM; Invitrogen)-based assay, as previously described [17]. Biotin conjugation was confirmed using a dot blot assay: $1 \mu \mathrm{g}$ of each PS-PEG or PS-PEG-biotin bead was blotted onto a nitrocellulose membrane, blocked with 5\% nonfat milk in PBS-Tween 20 (PBS-T) for $1 \mathrm{~h}$ at room temperature, incubated with streptavidin-horseradish peroxidase (SA-HRP, 1:5000 dilution) for 1 $\mathrm{h}$ at room temperature, and then detected using an ECL kit (BioRad, Hercules, CA, USA) and a FluorChemE imaging system (Protein Simple, San Jose, CA, USA).

\subsection{Cell culture and cell uptake assay}

Human monocytic THP-1, Raji B cell lymphoma, and Jurkat T-acute lymphoblastic leukemia cells were obtained from the University of North Carolina at Chapel Hill Tissue Culture Facility; no cell line authentication was performed after the cells were obtained. The Raji and Jurkat cells were maintained at 1 x $10^{6}$ cells/mL in RPMI 1640 medium containing $10 \%$ fetal bovine serum and $1 \mathrm{X}$ penicillin-streptomycin, with incubation at $37^{\circ} \mathrm{C}$ and $5 \% \mathrm{CO}_{2}$, and the cells used in the in vitro and in vivo experiments were cultured for 3-10 passages after thawing. The anti-CD20 $(\alpha \mathrm{CD} 20)$ fusion proteins $\left(1 \mathrm{~F} 5(\mathrm{scFv})_{4} \mathrm{SA}\right.$, B9E9 $\left.(\mathrm{scFv})_{4} \mathrm{SA}\right)$ and anti-TAG72 ( $\left.\alpha \mathrm{TAG} 72\right)$ fusion proteins $\left(\mathrm{CC} 49(\mathrm{scFv})_{4} \mathrm{SA}\right)$ were produced based on previously developed fusion gene constructs and purified according to a previously described method.[1822] . Briefly, the fusion proteins were individually expressed in the periplasmic space of Escherichia coli, spontaneously forming stable soluble tetramers of $174 \mathrm{kDa}$ molecular weight. The fusion proteins were 
formulated at a concentration of 2.5 or $5.0 \mathrm{mg} / \mathrm{mL}$ in phosphate buffer saline (PBS) containing $5 \%$ sorbitol and stored at $-20^{\circ} \mathrm{C}$ until use.

For the phagocytic uptake studies, THP-1 cells seeded into 24 well plates at $1.70 \times 10^{5}$ cells $/ \mathrm{mL}$ were differentiated in culture medium containing $200 \mathrm{nM}$ phorbol 12-myristate 13-acetate (PMA; SigmaAldrich, St. Louis, MO, USA) [17]. The PMA-containing medium was removed 3 days later and replaced with fresh culture medium, followed by incubation with various PS-PEG-biotin particles at a 1:10 cell:particle ratio for $12 \mathrm{~h}$. For the lypmhoma cell uptake studies, 1 x $10^{5}$ cells were seeded into 96-well plates and incubated with $500 \mathrm{nM}$ fusion protein (FP) for $4 \mathrm{~h}$. After washing to remove unbound FP, the cells were then incubated with various PS-PEG-biotin beads for $12 \mathrm{~h}$ at a $10^{4}: 1$ particle:cell ratio. Flow cytometry was performed using a FACSCanto instrument (BD, Franklin Lakes, NJ, USA). At least 10,000 events were recorded per sample, and the data were analyzed using BD FACSDiva software. The data represent $\mathrm{n}=3$ independent experiments performed in triplicate.

\subsection{Pharmacokinetics and biodistribution of biotinylated nanoparticles}

Female BALB/c mice (19-24 g body weight) were obtained from Charles River Laboratories (Wilmington, MA, USA), and all animal experiments carried out in accordance with an animal use protocol (\#14-098) approved by the University of North Carolina Animal Care and Use Committee. The mice were injected with fully modified PS-PEG-biotin nanoparticles $(15 \mathrm{mg} / \mathrm{kg}$, i.v.) in a total of $100 \mu \mathrm{L}$ of PBS via the tail vein, and the mice were sacrificed at various time points $(0,1,2$, and $6 \mathrm{~h} ; \mathrm{n} \geq 3$ mice randomized to each time point). Tissues (heart, liver, kidneys, spleen, lungs) and blood were collected, and the tissue biodistribution of the particles was determined using an IVIS Kinetic fluorescence imaging system with excitation at $465 \mathrm{~nm}$. The fluorescence of particles in the blood was measured using a SpectraMax 2 microplate reader and compared to a standard curve generated using green fluorescent PS beads added to untreated blood. PK analysis of the blood concentration data was conducted with PKSolver using a one-compartment model fit to the data [23, 24]. 


\subsection{Biodistribution of pre-targeted nanoparticles in mouse models containing single and dual tumors}

Female athymic nude mice (20-24 g body weight) were obtained from the University of North Carolina at Chapel Hill Small Animal Core, and all animal experiments carried out in accordance with an animal use protocol (\#14-054) approved by the University of North Carolina Animal Care and Use Committee. For single tumor-bearing mice, the animals were inoculated with Raji cells ( $2.5 \times 10^{7}$ cells each) on the right flank, and, for dual tumor-bearing mice, the animals were also inoculated with Jurkat cells $\left(2.5 \times 10^{7}\right.$ cells each) on the left flank. The mice were maintained on a biotin-free diet (Harlan Laboratories, Indianapolis, IN, USA) for at least $7 \mathrm{~d}$ prior to the start of the biodistribution studies. After the tumors reached $\geq 100$ $\mathrm{mm}^{3}$ in size, $\alpha \mathrm{CD} 20$ and/or $\alpha \mathrm{TAG} 72 \mathrm{FP}(250 \mu \mathrm{g}$ each) were administered i.v., followed by PS-PEGbiotin particles ( $15 \mathrm{mg} / \mathrm{kg}$, i.v.) after $24 \mathrm{~h}$ ( $\mathrm{n} \geq 4$ mice randomized to each group). After another $24 \mathrm{~h}$, the mice were sacrificed, and tissues (heart, liver, spleen, lungs, kidneys, tumors) were collected. The tissues from treated and untreated animals were imaged using an IVIS Kinetic fluorescence imaging system with excitation at $465 \mathrm{~nm}$. The fluorescent signal present in the tissues was calculated as a percentage of the total recovered fluorescence for the collected tissue samples.

\subsection{Statistical analysis}

Group comparisons were performed using one-way ANOVA, followed by Dunnett's post hoc test, on SAS 9.3 software. A p-value $<0.05$ was considered to indicate statistical significance. All data are presented as mean $\pm \mathrm{SD}$.

\section{Results}

\subsection{Synthesis and characterization of PS-PEG-biotin}

Pretargeting molecules based on $\mathrm{scFv}$ chains linked to SA represent an appealing platform for proof-ofconcept studies due to the exceptionally high affinity between biotin and streptavidin. To formulate biotinylated nanoparticles for use with the SA-based pretargeting molecules, we prepared a series of 
densely PEGylated polystyrene beads ( 100 nm diameter) with different molar ratios of terminal biotin substitution, ranging from 0-100 mol\% of all grafted PEG chains. All particles ranged between $~ 110-140$ $\mathrm{nm}$ in average diameter (Figure 2A), exhibited nearly neutrally charged surfaces ( $\geq-10 \mathrm{mV}$ ) (Figure 2A), and possessed very dense PEG coverage $\left(\geq 2.0 \mathrm{PEG} / \mathrm{nm}^{2}\right)$ irrespective of the biotin density (Figure $2 \mathrm{~B}$ ). The total number of biotin groups per nanoparticle was estimated based on the number of grafted PEG chains and the input molar ratio of biotin PEG (Figure 2B); increasing biotin substitution at higher biotin PEG ratios was validated by a dot blot assay (Supplemental Figure 1).

Presentation of surface ligands at high densities typically result in increased phagocytic clearance of nanoparticles [25-27]. Although biotin is exceedingly small (244 Da) and is a naturally occurring molecule in biological systems, we decided to quantify the uptake of PS-PEG-biotin beads by differentiated human macrophage-like THP-1 cells in vitro. Similar to beads modified with only methoxy PEG (0 mol\% biotin), which are exceptionally resistant to uptake by phagocytic cells and exhibit prolonged circulation profiles in vivo [17], PS-PEG-biotin nanoparticles effectively evaded uptake by differentiated THP-1 cells across all biotin densities (Figure 2C). 

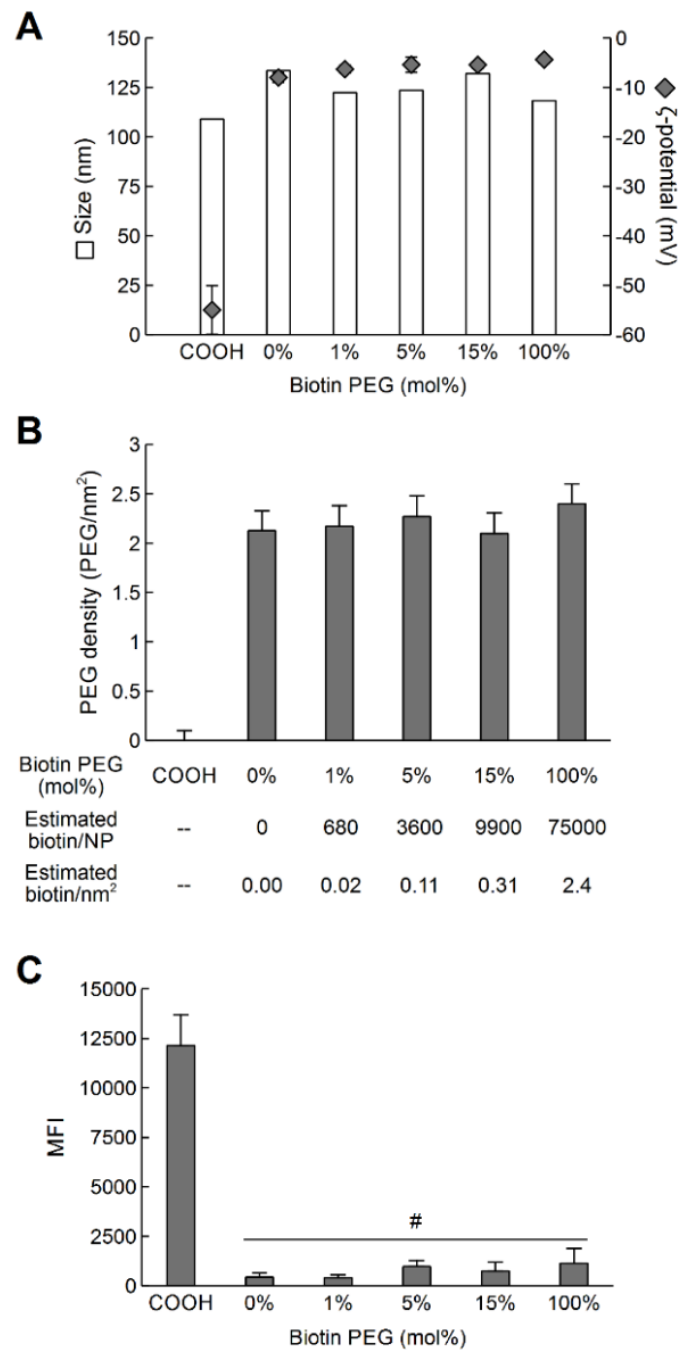

Figure 2. PS-PEG-biotin nanoparticle characterization. A) Hydrodynamic diameter (blue bars) and surface charge (diamonds) of unmodified PS beads $(\mathrm{COOH})$ and beads modified with varying mol\% of biotin PEG. B) Total PEG density of unmodified PS beads (COOH) and beads modified with varying mol\% of biotin PEG. C) Mean cellular fluorescence intensity (MFI) of differentiated human THP-1 cells incubated with PS-COOH, PS-PEG and PS-PEG-biotin beads. \# indicates $P<0.01$ vs. PS-COOH beads. The data represent $n \geq 2$ independent experiments performed in at least triplicate.

\subsection{Pretargeted delivery of PS-PEG-biotin nanoparticles in vitro}

To validate our hypothesis that pretargeting cells with different bispecific FPs can facilitate specific delivery of nanoparticles to distinct lymphoma cells, we measured the binding of various PS-PEG-biotin nanoparticles with different levels of biotin substitution to Raji (B-lymphoma; CD20 ${ }^{+} / \mathrm{TAG}^{-}$) and 
Jurkat (T-lymphoma; CD20־/TAG72 ${ }^{+}$) cells pretargeted with bispecific streptavidin-based fusion proteins containing scFvs against CD20 or TAG72. In both cell lines, when pretargeted with the corresponding FP, increasing surface biotin density improved nanoparticle binding (Figure 3). In contrast, no significant differences in nanoparticle binding were observed between particles of varying biotin density, even at the highest biotin densities, in the absence of FP and when pretargeted with control FP (i.e., $\alpha$ TAG72 FP for Raji cells and $\alpha$ CD20 FP for Jurkat cells). Pretargeting of Raji cells with $\alpha$ CD20 FP resulted in a 15fold greater uptake of fully biotinylated PS-PEG-biotin nanoparticles (100 mol\% biotin) compared to methoxy PEG-coated nanoparticles without biotin functionalization (i.e., PS-PEG; $p<0.01$ ), as well as 9- and 6- fold greater nanoparticle uptake than with no FP or pretargeting using $\alpha$ TAG72 FP, respectively $(p<0.01)$. Similarly, in Jurkat cells, pretargeting with $\alpha$ TAG72 FP enhanced the binding of fully biotinylated PS-PEG-biotin nanoparticles by $~ 18$-fold compared to PS-PEG nanoparticles without biotin $(p<0.01$ ), as well as by $\sim 8$ - and 6-fold relative to cells pretreated with no FP or $\alpha \mathrm{CD} 20 \mathrm{FP}$, respectively $(p<0.01)$. Compared to nanoparticles with lower biotin substitution, the fully biotinylated PS-PEG-biotin nanoparticles exhibited the greatest uptake with pretargeting yet were resistant to uptake by macrophage-like THP-1 cells in vitro; thus, we proceeded with subsequent in vivo studies using fully biotinylated PS-PEG-biotin nanoparticles. 

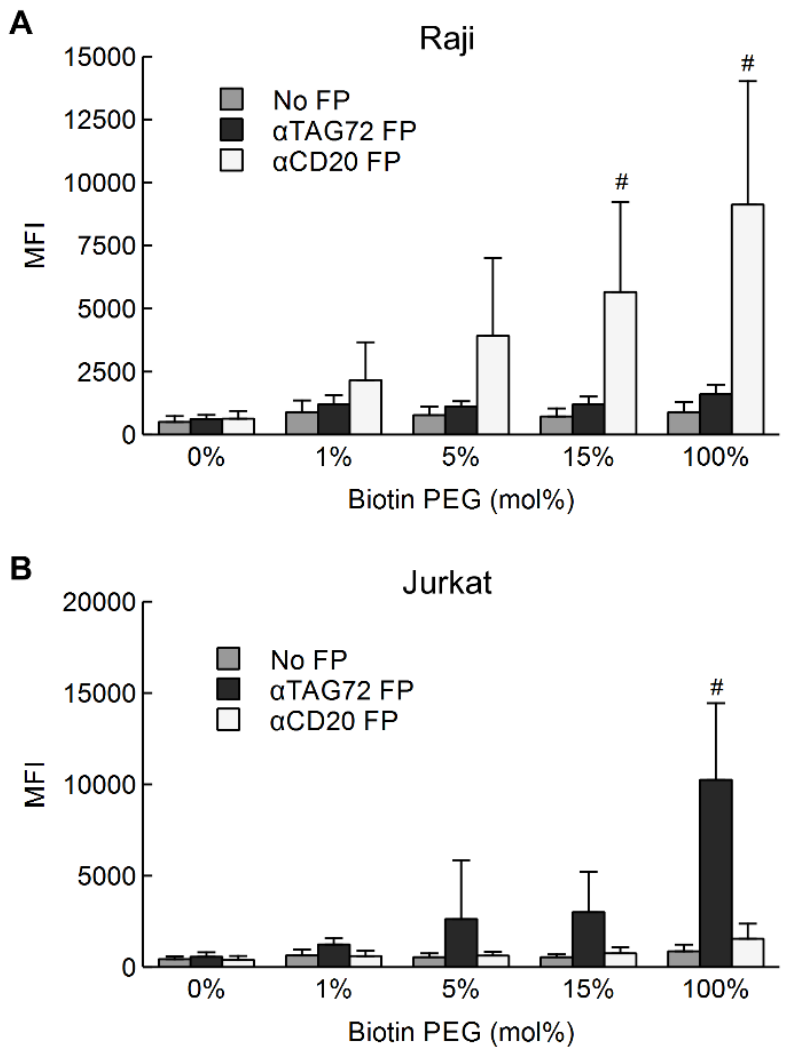

Figure 3. A) Raji and B) Jurkat cells preincubated with or without CD20- or TAG72-specific pretargeting fusion proteins (FPs) for $4 \mathrm{~h}$, followed by incubation with various PS-PEG-biotin beads for $12 \mathrm{~h}$. The mean cellular fluorescence intensity (MFI) was quantified using flow cytometry. All data represent at least $\mathrm{n}=3$ independent experiments performed in triplicate. \# indicates $\mathrm{P}<0.01$ vs. no FP group and control FP group.

\subsection{PS-PEG-biotin circulation kinetics and tissue biodistribution}

Since effective accumulation of nanoparticles in tumor tissue is thought to be highly dependent on the extended circulation times in the blood, we evaluated the circulation kinetics and tissue biodistribution of fully biotinylated PS-PEG-biotin nanoparticles in normal BALB/c mice. Interestingly, despite the demonstrated resistance to macrophage uptake in vitro (Figure 2C), we observed very rapid elimination of the PS-PEG-biotin nanoparticles from the blood (Figure 4A), with a circulation half-life of only $~ 40$ min. Similar to most nanoparticle systems, the liver, along with the spleen, represents the primary organ of nanoparticle disposition, both in relative (\%ID/g) and absolute (\%ID) quantities (Figure 4B). Although the in vivo pharmacokinetic profile of the fully biotinylated PS-PEG-biotin particles did not reflect 
prolonged circulation that was expected based on the in vitro THP-1 uptake results (Figure 2C) or the extended circulation kinetics of similar nanoparticles modified with only methoxy-PEG (e.g., $\mathrm{t}_{1 / 2} \sim 14 \mathrm{~h}$ ) [17], we nevertheless proceeded to evaluate the tumor targeting potential of pretargeting in vivo using the fully biotinylated PS-PEG-biotin nanoparticles due to the high cell-specific uptake observed in vitro.

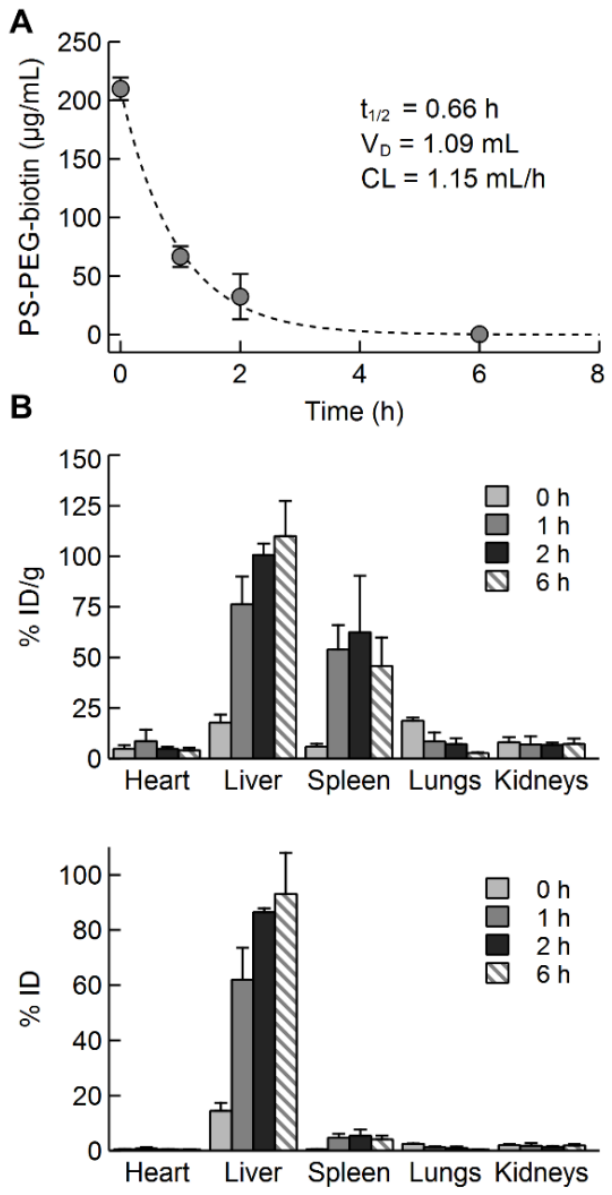

Figure 4. A) The blood circulation profile of PS-PEG-biotin (100 mol\%) particles in normal BALB/c mice $(n \geq 3)$. The dashed line represents the fit for a one-compartment model used to calculate the circulatory half-life $\left(\mathrm{t}_{1 / 2}\right)$, volume of distribution $\left(\mathrm{V}_{\mathrm{D}}\right)$, and clearance $(\mathrm{CL})$. B) Tissue biodistribution of fully biotinylated PS-PEG-biotin at various time points after i.v. injection. Injected dose/g (\%ID/g; top) and injected dose (\%ID; bottom) was quantified from the 2D fluorescent organ image signal intensities. All data represent the mean \pm SD of at least $n=3$ animals. 


\subsection{Biodistribution and tumor accumulation of pretargeted PS-PEG-biotin nanoparticles in single tumor}

mouse model

To determine whether pretargeting could enable specific delivery of biotinylated nanoparticles to tumors in spite of the poor circulation kinetics of the PS-PEG-biotin (100 mol\%) beads, we dosed single Raji tumor-bearing mice with $250 \mu \mathrm{g}$ of $\alpha \mathrm{CD} 20$ or $\alpha \mathrm{TAG} 72 \mathrm{FP}$ and then, $24 \mathrm{~h}$ later, administered $15 \mathrm{mg} / \mathrm{kg}$ of PS-PEG-biotin nanoparticles. Pretargeting with $\alpha$ CD20 FP significantly increased the amount of nanoparticles distributed in the Raji tumor $(p<0.05)$, with a $\sim 4$-fold increase in the tumor $\% \mathrm{ID} / \mathrm{g}$ compared to no FP and control $\alpha$ TAG72 FP (Figure 5A). Prior administration of $\alpha \mathrm{CD} 20$ or $\alpha \mathrm{TAG} 72$ did not significantly affect the biodistribution of PS-PEG-biotin beads to normal organs, and the nanoparticles were mainly distributed in the liver and spleen, as is common for the clearance of particles $\sim 100 \mathrm{~nm}$ in size (Figure 5B). In agreement with the short circulation half-life observed in BALB/c mice, no particles were detectable in circulation when the animals were sacrificed at $24 \mathrm{~h}$ post-injection (data not shown).
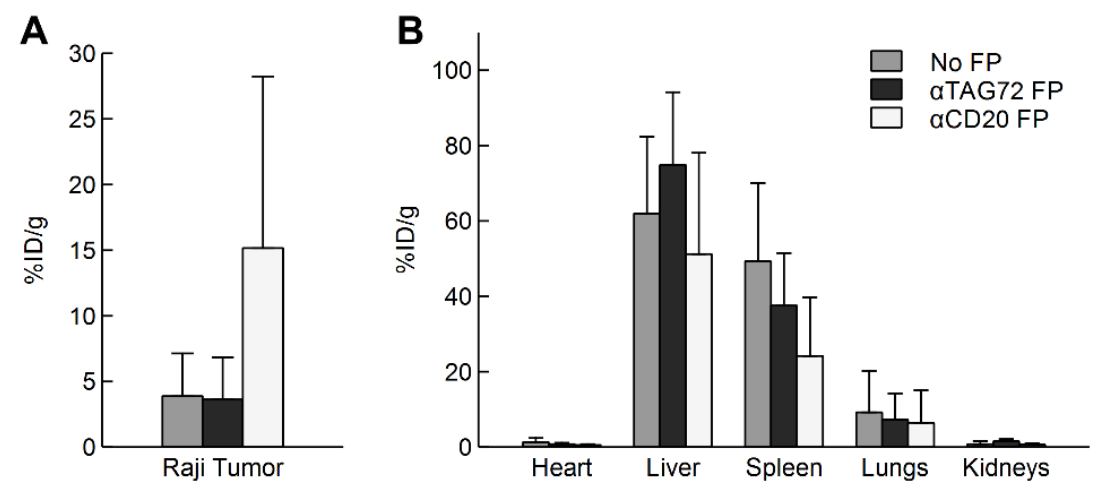

Figure 5. A) Raji tumor biodistribution and B) normal organ biodistribution of pretargeted PS-PEGbiotin at $24 \mathrm{~h}$. Tumor cells were pretargeted with $\alpha \mathrm{CD} 20$ or $\alpha \mathrm{TAG} 72$ FPs ( $250 \mu \mathrm{g}$ each), followed by nanoparticle injection after $24 \mathrm{~h}$. All data represent the mean \pm SD of at least $\mathrm{n}=4$ animals. Tissue injected dose/g (\% ID/g; top) was quantified from the 2D fluorescent organ image signal intensities. * indicates $p<0.05$ vs. no FP group. 


\subsection{Biodistribution and tumor accumulation of pretargeted PS-PEG-biotin nanoparticles in dual tumor}

\section{mouse model}

Despite the convenience and ease of xenograft models, a major shortcoming with tumor xenografts is that the animals are typically inoculated with a homogenous population of cancer cells with an identical genetic background, which naturally suppresses delivery challenges associated with tumor heterogeneity. To evaluate pretargeted nanoparticle delivery to distinct tumor cell populations in vivo, we decided to inoculate athymic nude mice with Raji and Jurkat cells on the right and left flanks of the same mice, respectively, to generate a dual tumor xenograft model. The animals were then given $250 \mu \mathrm{g}$ each of $\alpha \mathrm{CD} 20$ and/or $\alpha$ TAG72 FP, followed by $15 \mathrm{mg} / \mathrm{kg}$ of PS-PEG-biotin nanoparticles after $24 \mathrm{~h}$. The use of FP pretargeting increased the amount of particles found in both the Raji and Jurkat tumors (Figure 6A), with the combination FP group exhibited $\sim 4$-fold higher tumor accumulation compared to the no FP group for Raji tumors $(p<0.05)$. While the combined use of $\alpha \mathrm{CD} 20$ and $\alpha$ TAG72 FPs appeared to increase PS-PEG-biotin present in Jurkat tumors as well, no statistically differences were found between groups, likely due to high mouse-to-mouse tumor variability. The extent of nanoparticle accumulation, as measured by $\% \mathrm{ID} / \mathrm{g}$, was substantially different between the Raji and Jurkat tumors, with markedly higher accumulation in Raji vs. Jurkat tumors $(3.5 \% \mathrm{ID} / \mathrm{g}$ and $12.5 \% \mathrm{ID} / \mathrm{g}$ vs. $1.4 \% \mathrm{ID} / \mathrm{g}$ and $4.4 \% \mathrm{ID} / \mathrm{g}$ without FP and with combined FP, respectively), likely reflecting differences in tumor physiology and extravasation of the nanoparticles. Similar to the results above with single tumor-bearing mice, pretargeting did not significantly alter the biodistribution of the particles to normal organs compared to the no FP control (Figure 6B); the vast majority of particles were found in the liver and spleen, and the nanoparticles were completely eliminated from systemic circulation by $24 \mathrm{~h}$ (data not shown). 

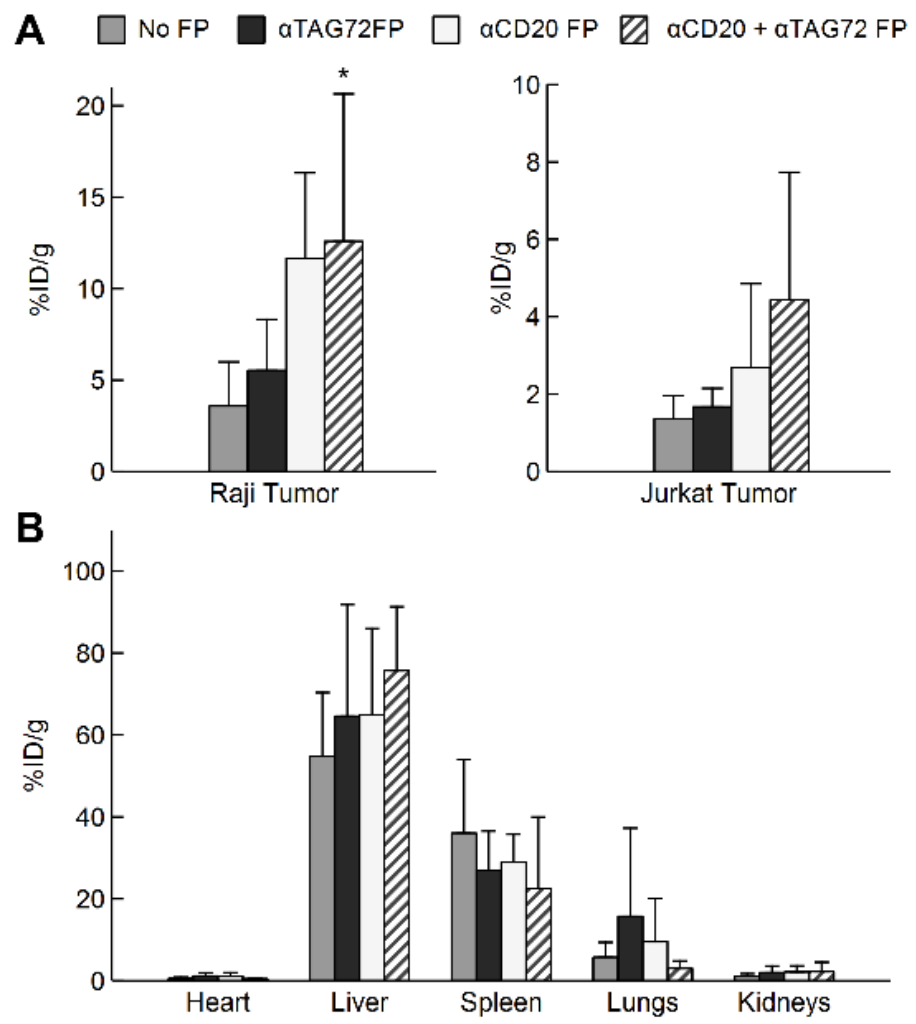

Figure 6. A) Raji (left) and Jurkat (right) tumor biodistribution and B) normal organ biodistribution of pretargeted PS-PEG-biotin at $24 \mathrm{~h}$. Tumor cells were pretargeted with $\alpha \mathrm{CD} 20$ and/or $\alpha$ TAG72 FPs (250 $\mu \mathrm{g}$ each), followed by nanoparticle injection after $24 \mathrm{~h}$. All data represent the mean \pm SD of at least $\mathrm{n}=4$ animals.

\section{Discussion}

The complexity of the tumor physiology and its microenvironment, including inter- and intratumoral heterogeneity, necessitates the exploration of alternative, multi-faceted strategies that can more effectively deliver therapeutics to the full spectrum of tumor cells than can be typically achieved with single ligand-conjugated nanoparticles. Notably, many of the shortcomings of common delivery systems for chemotherapy are the same deficiencies associated with conventional radioimmunotherapy.

Nevertheless, despite the promise and early success of PRIT, the use of pretargeting molecules to enhance nanoparticle delivery has remained largely underexplored to date. The few studies that have investigated pretargeting of nanoparticles to cancer cells [14, 28-32] all evaluated delivery to a homogeneous cell 
population in vivo, or relied on multiple unique pairs of pretargeting molecules and nanoparticles for detection of different cancer cells. This overlooks one of the primary advantages to pretargeting: by decoupling cellular targeting from nanoparticle design/formulation, the same nanoparticles can easily be targeted to multiple different cells by simply tuning the pretargeting molecule(s) used. Here, in good agreement with theory, we demonstrated that pretargeting can enhance cellular delivery of a single nanoparticle formulation to at least two molecularly distinct lymphoma cells in vitro and in vivo. Although many further improvements are needed, we believe the pretargeting approach, precipitated by the convergence of biotechnology, nanotechnology and drug delivery, represents a promising strategy that could more effectively deliver therapeutics to diverse cell populations. Improved therapeutics delivery to different cells simultaneously may in turn lead to more durable suppression or elimination of the residual tumor while minimizing eventual recurrence and/or the development of drug-resistant tumors.

The origins of the pretargeting concept is rooted in the pioneering work of Press and colleagues, who widely explored pretargeting as a strategy to overcome the shortcomings of conventional radioimmunotherapy, in particular the non-specific deposition of radiolabeled MAbs in normal organs that result in low tumor-specific delivery of radiation and significant toxicity [13]. By utilizing bispecific proteins that are non-radioactive, along with radiolabeled effector molecules that can be rapidly cleared, PRIT significantly improved the therapeutic index of radioisotope treatment compared to conventional radioimmunotherapy [13, 33, 34] and increased the maximum tolerated dose for radionuclides [35]. Pretargeting has been broadly tested for a variety of blood malignancies in vivo including leukemia (CD45), B cells (CD20, HLA-DR and CD22) and multiple myeoloma (CD38), achieving tumor-to-blood ratios ranging from 2:1 to $638: 1$, and markedly improved survival rates [36-42]. These encouraging results in animal models have translated into clinical studies of PRIT, which to date have yielded promising results with reasonable tumor response rates and limited toxicity in phase I/II trials [43, 44].

The extent of nanoparticle targeting to the B- and T- lymphoma tumors in our mouse model remain limited relative to other organs, particularly the liver and spleen. One possible reason is inadequate accumulation of the pretargeting molecules within the tumor milieu $[18,45]$. When coupled 
with the relatively weak affinity between scFvs and the tumor, this likely results in limited binding and retention of extravasated pretargeting molecules on cancer cells in the tumor and, consequently, limited capture of the nanoparticles by bound FP on cancer cells. For both single and dual tumor-bearing mice, we observed an increase in nanoparticle tumor accumulation with $\alpha \mathrm{CD} 20 \mathrm{FP}$, even in the case of CD20Jurkat tumors, suggesting that more $\alpha \mathrm{CD} 20 \mathrm{FP}$ may have extravasated into and remained at the tumor site compared to $\alpha$ TAG72 FP. This nonspecifically accumulation of pretargeting FP in the tumor tissue may have agglutinated subsequently dosed nanoparticles that extravasated into the tumor, thus improving nanoparticle retention rather than enabling specific homing and binding to target cancer cells.

We had initially hypothesized that the presence of biotin on the surface of nanoparticles would not compromise the prolonged circulation of densely biotinylated PEGylated nanoparticles. The hypothesis was based in part on the low MW of biotin relative to the MW of PEG and the effective pretargeting observed with PRIT using biotinylated radiolabels, and was supported by the limited uptake by macrophage-like cells in vitro. Nevertheless, fully biotinylated nanoparticles were quickly eliminated in mice; at 6 hours post-injection, and there was no detectable amount of PS-PEG-biotin nanoparticles in the blood compared to $~ 70 \%$ of the injected dose for previously evaluated PS-PEG-methoxy nanoparticles [17]. The poor circulation kinetics likely limited the fraction of the injected nanoparticle dose that could reach and extravasate at the tumor site. Since biotin is a naturally occurring vitamin, we speculate that the rapid accumulation of biotin-modified nanoparticles in the liver, which functions as a reservoir for biotin, can probably be attributed the presence of vitamin receptors in the liver and on MPS cells $[46,47]$ combined with the highly multivalent nature of biotin presentation on nanoparticles. Together, this likely led to more efficient capture of nanoparticles decorated with multiple biotin groups than radioisotopes functionalized with a single biotin group. The poor nanoparticle circulation kinetics might be partially resolved by lowering the biotin density on the nanoparticle surface, thus widening the time window during which the particles can circulate and permeate into tumors. Nevertheless, reduced surface biotin density would also decrease the binding rate of nanoparticles to the pretargeting FPs 
present on the cell surface and in the tumor site. Instead, we expect the use of alternative binding pairs (i.e., nanoparticle-pretargeting molecule linkages) would avoid the problematic use of biotinylation and improve nanoparticle circulation and extravasation, as well as overcome immunogenicity commonly observed with streptavidin. However, the likely reduction in nanoparticle-pretargeting FP affinity (relative to that of streptavidin-biotin bonds) would reduce the efficiency of pretargeting molecules crosslinking nanoparticles to target cells. In our opinion, the development of pretargeting molecules with high binding affinities to both cells and nanoparticles and bio-inert binding pairs represent the most critical challenge for future development of pretargeted delivery of nanoparticles.

The use of multiple pretargeting molecule combinations have been previously evaluated for PRIT of lymphoma with variable expression of CD20, CD22, and HLA-DR [39, 48]. Interestingly, the authors found that a single pretargeting molecule treatment provided superior tumor-to-normal organ ratios and survival rates, whereas a cocktail of three different pretargeting molecules (against CD20, CD22 and HLA-DR) resulted in increased liver biodistribution of the radioactive payload and increased toxicity. In contrast, pretargeting with $\alpha \mathrm{CD} 20$ and/or $\alpha$ TAG72 FPs did not alter nanoparticle biodistribution to normal organs in single and dual tumor-bearing mice in our study (Figure 6). This disparity in the effects of extraneous pretargeting molecules most likely resulted from differences in the features and properties of the therapeutic agents used. Unlike the small-molecule radionuclide carriers used in the PRIT studies, the majority of systemically dosed nanoparticles are eventually eliminated by MPS organs such as the liver and spleen, which may eclipse any changes in biodistribution caused by mismatched pretargeting FP administration. Nevertheless, the potential effects of pretargeting molecules cocktails on nanoparticle biodistribution and toxicity should be further evaluated in the future, particularly for different pretargeting molecule/nanoparticle combinations.

\section{Conclusions}

Tumor heterogeneity presents a complex delivery challenge, as nanoparticle drug carriers must accumulate in tumor tissue while also maximally targeting the diverse cell populations present within a 
given tumor and/or patient. Here, we explored the simultaneous use of multiple pretargeting bispecific fusion proteins to enhance nanoparticle delivery to heterogeneous target cell populations. We found that increasing amounts of surface biotin on the nanoparticles enabled highly specific cell uptake by tumor cells pretargeted by corresponding fusion proteins in vitro, and, for single tumor-bearing mice, pretargeting with tumor-specific fusion protein also significantly improved nanoparticle tumor accumulation even in the face of the poor circulation kinetics of the biotinylated nanoparticles. Additionally, in dual tumor-bearing mice, the use of pretargeting with a fusion protein cocktail directly increased nanoparticle delivery to both tumor types. Our findings support further investigations into the use of pretargeting fusion protein cocktails as an alternative targeting strategy to enhance nanoparticle delivery to a diverse array of molecularly distinct target cells.

Funding: This work was supported by the PhRMA Foundation Predoctoral Fellowship and UNC Dissertation Completion Fellowship (Q.Y.), the National Science Foundation Graduate Research Fellowship Program (C.L.P., DGE-1144081), The David and Lucile Packard Foundation (2013-39274, S.K.L.), The UNC Research Opportunities Initiative (S.K.L.), Carolina Center for Cancer Nanotechnology Excellence (U54CA151652) Pilot Grants Program (S.I.P. and S.K.L.), and startup funds from the Eshelman School of Pharmacy and Lineberger Comprehensive Cancer Center (S.K.L.). 


\section{References}

[1] J. Fang, H. Nakamura, H. Maeda, The EPR effect: Unique features of tumor blood vessels for drug delivery, factors involved, and limitations and augmentation of the effect, Adv Drug Deliv Rev, 63 (2011) 136-151.

[2] V.P. Torchilin, Passive and active drug targeting: drug delivery to tumors as an example, Handb Exp Pharmacol, (2010) 3-53.

[3] H. Maeda, Toward a full understanding of the EPR effect in primary and metastatic tumors as well as issues related to its heterogeneity, Adv Drug Deliv Rev, (2015).

[4] S.N. Ekdawi, J.M. Stewart, M. Dunne, S. Stapleton, N. Mitsakakis, Y.N. Dou, D.A. Jaffray, C. Allen, Spatial and temporal mapping of heterogeneity in liposome uptake and microvascular distribution in an orthotopic tumor xenograft model, J Control Release, 207 (2015) 101-111.

[5] V. Torchilin, Tumor delivery of macromolecular drugs based on the EPR effect, Adv Drug Deliv Rev, 63 (2011) 131-135.

[6] N. Bertrand, J. Wu, X. Xu, N. Kamaly, O.C. Farokhzad, Cancer nanotechnology: the impact of passive and active targeting in the era of modern cancer biology, Adv Drug Deliv Rev, 66 (2014) 2-25.

[7] D.B. Kirpotin, D.C. Drummond, Y. Shao, M.R. Shalaby, K. Hong, U.B. Nielsen, J.D. Marks, C.C. Benz, J.W. Park, Antibody Targeting of Long-Circulating Lipidic Nanoparticles Does Not Increase Tumor Localization but Does Increase Internalization in Animal Models, Cancer Res, 66 (2006) 67326740.

[8] Y.H. Bae, K. Park, Targeted drug delivery to tumors: Myths, reality and possibility, J Control Release, 153 (2011) 198-205.

[9] C.P. Leamon, S.R. Cooper, G.E. Hardee, Folate-Liposome-Mediated Antisense Oligodeoxynucleotide Targeting to Cancer Cells: Evaluation in Vitro and in Vivo, Bioconjug Chem, 14 (2003) 738-747.

[10] P.L. Bedard, A.R. Hansen, M.J. Ratain, L.L. Siu, Tumour heterogeneity in the clinic, Nature, 501 (2013) 355-364.

[11] M. Jamal-Hanjani, S.A. Quezada, J. Larkin, C. Swanton, Translational implications of tumor heterogeneity, Clin Cancer Res, 21 (2015) 1258-1266.

[12] D.M. Goldenberg, C.H. Chang, E.A. Rossi, W. J, McBride, R.M. Sharkey, Pretargeted molecular imaging and radioimmunotherapy, Theranostics, 2 (2012) 523-540.

[13] R.B. Walter, O.W. Press, J.M. Pagel, Pretargeted radioimmunotherapy for hematologic and other malignancies, Cancer Biother Radiopharm, 25 (2010) 125-142.

[14] Q. Yang, C.L. Parker, J.D. McCallen, S.K. Lai, Addressing challenges of heterogeneous tumor treatment through bispecific protein-mediated pretargeted drug delivery, J Control Release, (2015).

[15] A. Forero, P.L. Weiden, J.M. Vose, S.J. Knox, A.F. LoBuglio, J. Hankins, M.L. Goris, V.J. Picozzi, D.B. Axworthy, H.B. Breitz, R.B. Sims, R.G. Ghalie, S. Shen, R.F. Meredith, Phase 1 trial of a novel anti-CD20 fusion protein in pretargeted radioimmunotherapy for B-cell non-Hodgkin lymphoma, Blood, 104 (2004) 227-236.

[16] A. Forero-Torres, S. Shen, H. Breitz, R.B. Sims, D.B. Axworthy, M.B. Khazaeli, K.H. Chen, I. Percent, S. Besh, A.F. LoBuglio, R.F. Meredith, Pretargeted radioimmunotherapy (RIT) with a novel anti-TAG-72 fusion protein, Cancer Biother Radiopharm, 20 (2005) 379-390.

[17] Q. Yang, S.W. Jones, C.L. Parker, W.C. Zamboni, J.E. Bear, S.K. Lai, Evading immune cell uptake and clearance requires PEG grafting at densities substantially exceeding the minimum for brush conformation, Mol Pharm, 11 (2014) 1250-1258.

[18] Y. Lin, J.M. Pagel, D. Axworthy, A. Pantelias, N. Hedin, O.W. Press, A Genetically Engineered Anti-CD45 Single-Chain Antibody-Streptavidin Fusion Protein for Pretargeted Radioimmunotherapy of Hematologic Malignancies, Cancer Res, 66 (2006) 3884-3892.

[19] J. Gunn, S.I. Park, O. Veiseh, O.W. Press, M. Zhang, A pretargeted nanoparticle system for tumor cell labeling, Mol Biosyst, 7 (2011) 742-748.

[20] S.I. Park, J. Shenoi, J.M. Pagel, D.K. Hamlin, D.S. Wilbur, N. Orgun, A.L. Kenoyer, S. Frayo, A. Axtman, T. Bäck, Y. Lin, D.R. Fisher, A.K. Gopal, D.J. Green, O.W. Press, Conventional and pretargeted 
radioimmunotherapy using bismuth-213 to target and treat non-Hodgkin lymphomas expressing CD20: a preclinical model toward optimal consolidation therapy to eradicate minimal residual disease, Blood, 116 (2010) 4231-4239.

[21] J.M. Pagel, Y. Lin, N. Hedin, A. Pantelias, D. Axworthy, D. Stone, D.K. Hamlin, D.S. Wilbur, O.W. Press, Comparison of a tetravalent single-chain antibody-streptavidin fusion protein and an antibodystreptavidin chemical conjugate for pretargeted anti-CD20 radioimmunotherapy of B-cell lymphomas, Blood, 108 (2006) 328-336.

[22] J. Schultz, Y. Lin, J. Sanderson, Y. Zuo, D. Stone, R. Mallett, S. Wilbert, D. Axworthy, A tetravalent single-chain antibody-streptavidin fusion protein for pretargeted lymphoma therapy, Cancer Res, 60 (2000) 6663-6669.

[23] Q. Yang, S.W. Jones, C.L. Parker, W.C. Zamboni, J.E. Bear, S.K. Lai, Evading Immune Cell Uptake and Clearance Requires PEG Grafting at Densities Substantially Exceeding the Minimum for Brush Conformation, Mol Pharm, (2014).

[24] Y. Zhang, M. Huo, J. Zhou, S. Xie, PKSolver: An add-in program for pharmacokinetic and pharmacodynamic data analysis in Microsoft Excel, Comput Methods Programs Biomed, 99 (2010) 306314.

[25] J.R. Beech, S.J. Shin, J.A. Smith, K.A. Kelly, Mechanisms for targeted delivery of nanoparticles in cancer, Curr Pharm Des, 19 (2013) 6560-6574.

[26] Z. Cheng, A. Al Zaki, J.Z. Hui, V.R. Muzykantov, A. Tsourkas, Multifunctional Nanoparticles: Cost Versus Benefit of Adding Targeting and Imaging Capabilities, Science, 338 (2012) 903-910.

[27] F. Gu, L. Zhang, B.A. Teply, N. Mann, A. Wang, A.F. Radovic-Moreno, R. Langer, O.C. Farokhzad, Precise engineering of targeted nanoparticles by using self-assembled biointegrated block copolymers, Proc Natl Acad Sci U S A, 105 (2008) 2586-2591.

[28] M. Pulkkinen, J. Pikkarainen, T. Wirth, T. Tarvainen, V. Haapa-aho, H. Korhonen, J. Seppälä, K. Järvinen, Three-step tumor targeting of paclitaxel using biotinylated PLA-PEG nanoparticles and avidinbiotin technology: Formulation development and in vitro anticancer activity, European Journal of Pharmaceutics and Biopharmaceutics, 70 (2008) 66-74.

[29] S.A. Longman, P.R. Cullis, L. Choi, G. de Jong, M.B. Bally, A two-step targeting approach for delivery of doxorubicin-loaded liposomes to tumour cells in vivo, Cancer Chemother Pharmacol, 36 (1995) 91-101.

[30] J.J. Mulvey, C.H. Villa, M.R. McDevitt, F.E. Escorcia, E. Casey, D.A. Scheinberg, Self-assembly of carbon nanotubes and antibodies on tumours for targeted amplified delivery, Nat Nanotechnol, 8 (2013) 763-771.

[31] L. Nobs, F. Buchegger, R. Gurny, E. Allemann, Biodegradable nanoparticles for direct or two-step tumor immunotargeting, Bioconjug Chem, 17 (2006) 139-145.

[32] T.W. Chu, J. Yang, R. Zhang, M. Sima, J. Kopecek, Cell surface self-assembly of hybrid nanoconjugates via oligonucleotide hybridization induces apoptosis, ACS Nano, 8 (2014) 719-730.

[33] J.M. Pagel, N. Hedin, K. Subbiah, D. Meyer, R. Mallet, D. Axworthy, L.J. Theodore, D.S. Wilbur, D.C. Matthews, O.W. Press, Comparison of anti-CD20 and anti-CD45 antibodies for conventional and pretargeted radioimmunotherapy of B-cell lymphomas, Blood, 101 (2003) 2340-2348.

[34] D.J. Green, J.M. Pagel, E.R. Nemecek, Y. Lin, A. Kenoyer, A. Pantelias, D.K. Hamlin, D.S. Wilbur, D.R. Fisher, J.G. Rajendran, A.K. Gopal, S.I. Park, O.W. Press, Pretargeting CD45 enhances the selective delivery of radiation to hematolymphoid tissues in nonhuman primates, Blood, 114 (2009) 1226-1235. [35] D.J. Green, J.M. Pagel, A. Pantelias, N. Hedin, Y. Lin, D.S. Wilbur, A. Gopal, D.K. Hamlin, O.W. Press, Pretargeted radioimmunotherapy for B-cell lymphomas, Clin Cancer Res, 13 (2007) 5598s-5603s. [36] J.M. Pagel, D.C. Matthews, A. Kenoyer, D.K. Hamlin, D.S. Wilbur, D.R. Fisher, A.K. Gopal, Y. Lin, L. Saganic, F.R. Appelbaum, O.W. Press, Pretargeted radioimmunotherapy using anti-CD45 monoclonal antibodies to deliver radiation to murine hematolymphoid tissues and human myeloid leukemia, Cancer Res, 69 (2009) 185-192.

[37] J.M. Pagel, A.L. Kenoyer, T. Back, D.K. Hamlin, D.S. Wilbur, D.R. Fisher, S.I. Park, S. Frayo, A. Axtman, N. Orgun, J. Orozco, J. Shenoi, Y. Lin, A.K. Gopal, D.J. Green, F.R. Appelbaum, O.W. Press, 
Anti-CD45 pretargeted radioimmunotherapy using bismuth-213: high rates of complete remission and long-term survival in a mouse myeloid leukemia xenograft model, Blood, 118 (2011) 703-711.

[38] J.M. Pagel, N. Orgun, D.K. Hamlin, D.S. Wilbur, T.A. Gooley, A.K. Gopal, S.I. Park, D.J. Green, Y. Lin, O.W. Press, A comparative analysis of conventional and pretargeted radioimmunotherapy of B-cell lymphomas by targeting CD20, CD22, and HLA-DR singly and in combinations, Blood, 113 (2009) 4903-4913.

[39] J.M. Pagel, A. Pantelias, N. Hedin, S. Wilbur, L. Saganic, Y. Lin, D. Axworthy, D.K. Hamlin, D.S. Wilbur, A.K. Gopal, O.W. Press, Evaluation of CD20, CD22, and HLA-DR targeting for radioimmunotherapy of B-cell lymphomas, Cancer Res, 67 (2007) 5921-5928.

[40] D.J. Green, N.N. Orgun, J.C. Jones, M.D. Hylarides, J.M. Pagel, D.K. Hamlin, D.S. Wilbur, Y. Lin, D.R. Fisher, A.L. Kenoyer, S.L. Frayo, A.K. Gopal, J.J. Orozco, T.A. Gooley, B.L. Wood, W.I.

Bensinger, O.W. Press, A preclinical model of CD38-pretargeted radioimmunotherapy for plasma cell malignancies, Cancer Res, 74 (2014) 1179-1189.

[41] K. Subbiah, D.K. Hamlin, J.M. Pagel, D.S. Wilbur, D.L. Meyer, D.B. Axworthy, R.W. Mallett, L.J. Theodore, P.S. Stayton, O.W. Press, Comparison of immunoscintigraphy, efficacy, and toxicity of conventional and pretargeted radioimmunotherapy in CD20-expressing human lymphoma xenografts, $\mathrm{J}$ Nucl Med, 44 (2003) 437-445.

[42] M. Zhang, Z. Zhang, K. Garmestani, J. Schultz, D.B. Axworthy, C.K. Goldman, M.W. Brechbiel, J.A. Carrasquillo, T.A. Waldmann, Pretarget radiotherapy with an anti-CD25 antibody-streptavidin fusion protein was effective in therapy of leukemia/lymphoma xenografts, Proc Natl Acad Sci U S A, 100 (2003) 1891-1895.

[43] C. Rousseau, F. Kraeber-Bodéré, J. Barbet, J.-F. Chatal, Pretargeted radioimmunotherapy: clinically more efficient than conventional radioimmunotherapy?, Eur J Nucl Med Mol Imaging, 40 (2013) 13731376.

[44] S.M. Larson, J.A. Carrasquillo, N.-K.V. Cheung, O.W. Press, Radioimmunotherapy of human tumours, Nat Rev Cancer, 15 (2015) 347-360.

[45] N.K. Cheung, S. Modak, Y. Lin, H. Guo, P. Zanzonico, J. Chung, Y. Zuo, J. Sanderson, S. Wilbert, L.J. Theodore, D.B. Axworthy, S.M. Larson, Single-chain Fv-streptavidin substantially improved therapeutic index in multistep targeting directed at disialoganglioside GD2, J Nucl Med, 45 (2004) 867877.

[46] J. Zempleni, D.M. Mock, Uptake and metabolism of biotin by human peripheral blood mononuclear cells, Am J Physiol, 275 (1998) C382-388.

[47] D.L. Vesely, S.F. Kemp, M.J. Elders, Isolation of a biotin receptor from hepatic plasma membranes, Biochem Biophys Res Commun, 143 (1987) 913-916.

[48] A. Pantelias, J.M. Pagel, N. Hedin, L. Saganic, S. Wilbur, D.K. Hamlin, D.S. Wilbur, Y. Lin, D.

Stone, D. Axworthy, A.K. Gopal, O.W. Press, Comparative biodistributions of pretargeted

radioimmunoconjugates targeting CD20, CD22, and DR molecules on human B-cell lymphomas, Blood, 109 (2007) 4980-4987. 